\title{
Plus End of the Actin Filament
}

National Cancer Institute

\section{Source}

National Cancer Institute. Plus End of the Actin Filament. NCI Thesaurus. Code C33332.

The end of an actin filament that is polymerizing at a faster rate. 